\title{
Editorial
}

\section{Palliative Care Ausbildung in Österreich - die Aufgabe Empathie und Wissenschaft zu verbinden - der 3. Intensivlehrgang Palliativmedizin}

\section{Training palliative care in Austria - empathy combined with science - the 3rd intensive course of palliative medicine}

\author{
Johannes G. Meran \\ Interne Abteilung, Krankenhaus der Barmherzigen Brüder Wien, Wien, Österreich
}

Die Hospizbewegung hat das wichtigste Ziel jeder ärztlichen Arbeit wieder neu in Erinnerung gerufen. Ärztliche Kunst ist begrenzt, doch die Begleitung und Bemühung um Lebensqualität darf nicht an medizintechnischen Grenzen stehen bleiben. Im Gegenteil, die größten Herausforderungen bestehen darin, menschliche Wärme und Empathie mit modernster Medizintechnik gerade in den existentiellen Situationen in Harmonie zu bringen. Fachkompetenz des Arztes bedeutet im Angesicht des Sterbenden auch den Mut zu haben, die Grenzen scheinbar ohnmächtig auszuhalten. Gerade dann nicht davonzulaufen, wenn alle medizinischen Bemühungen versagen, sondern die neuen Ziele der Begleitung physisch, psychisch, spirituell zu entdecken, Beschwerden kompetent zu lindern und zuletzt einfach den Patienten nicht alleine zu lassen. Denn nur wer die Erfahrung macht angenommen zu sein, wird sein Leid annehmen können.

Der 3. Universitätslehrgang Palliativmedizin hat sich wiederum der Aufgabe gestellt, interdisziplinäre Konzepte der Palliativmedizin in praxisorientierter Weise zu unterrichten. Die wissenschaftlich bewährte Methode des Lernens durch Patientengeschichten (case-reports), die unterschiedliche Situationen und Herausforderungen systematisch anhand eines konkreten Beispiels beleuchtet, erlaubt einen praxisnahen Zugang zu dem Thema. Es wird dabei besonders

Korrespondenz: Prim. Univ.-Prof. Dr. Johannes G. Meran, MA, Medizinische Abteilung, Krankenhaus der Barmherzigen Brüder Wien, Johannes von Gott Platz 1, 1020 Wien, Österreich.

Fax: ++43-1-21121-2103, E-Mail: johannes.meran@bbwien.at deutlich, wie komplex und interdisziplinär palliative Medizin ist - und fast immer sogar weit über die fachlich medizinische Ebene hinausgeht. Ein besonderes Anliegen der Kursleitung ist es, mit Unterstützung von inländischen und ausländischen Experten die neuesten wissenschaftlichen Erkenntnisse in die tägliche Praxis zu stellen. Die Aufbruchsstimmung - auch anspruchsvolle wissenschaftliche Fragestellungen neben der großen Stärke der Palliative Care im Sinne der psychosozialen Kompetenz einzubringen - ist in diesem Jahr sehr gut gelungen. Die vorliegenden Arbeiten sind ausgewählte Beispiele, die unseren Ansatz verdeutlichen.

Sinnhaftigkeit und Dauer einer Chemotherapie sind Fragen, die sich in der palliativen Chemotherapie früher oder später immer stellen und die Kenntnis der neuesten Studiendaten ebenso erfordern, wie eine einfühlsame Kommunikation mit den Patienten, um den richtigen therapeutischen Weg $\mathrm{zu}$ finden. Die Arbeit von Heidemarie Seemann geht dieser Frage unter dem Leitziel der Lebensqualität nach. Tumorschmerzen sind meist die wichtigste Störung der Lebensqualität. Trotz Einsatzes aller konventioneller Mitteln bleiben manchmal Extremsituationen, die nicht ausreichend behandelbar sind. In solchen Fällen ist die invasive Schmerztherapie eine hilfreiche Erweiterung unseres Spektrums, wie die Arbeit von Ofenschüssl zeigen kann. Interdisziplinarität ist Kennzeichen einer gut funktionierenden Palliativmedizin. Gerade die Strahlentherapie erlaubt Behandlungsansätze in schwierigen Situationen und ist die wichtigste Maßnahme bei 
Hirnmetastasen. Die Arbeit von Windischbauer erlaubt einen Einblick in solch eine Behandlungssituation. Weimann diskutiert ein weiteres palliatives Kernthema, die Ernährungstherapie in der letzten Lebensphase, aber auch die Grenzen der Möglichkeiten bei einem fortschreitenden malignen Grundleiden Gewicht und Kalorienzufuhr stabil zu halten. Wiederum geht es um das Ziel der Lebensqualität, die über einem mechanistischen Denken steht.

Dieses Ziel geht auch aus der Definition der World Health Organisation (WHO) hervor: „Palliativmedizin ist die aktive ganzheitliche Behandlung von Patienten mit einer progredienten, weit fortgeschrittenen Erkrankung und einer begrenzten Lebenserwartung zu einer Zeit, in der die Erkrankung nicht mehr auf eine kurative Behandlung anspricht und die Beherrschung der Schmerzen, andere Krankheitsbeschwerden, psychologischen, sozialen und spirituellen Problemen höchste Priorität besitzt“. Palliativmedizin als Wegbegleiter und bedarfsorientiertes Konzept, das sich dem Tempo und den Erfordernissen individuell anpassen muss. Palliativmedizin als ein Brückenfach, das sich einem interdisziplinären Ansatz verpflichtet weiß und im Interesse des Patienten Grenzen von Fachgebieten ebenso überschreitet, wie konventionelle Behandlungskriterien. Der Bogen spannt sich von empathischer Hospizpflege bis hin zu einer individualisierten, aber hochmodernen Palliativmedizin im engeren Sinne. Der Universitätslehrgang versucht, diesen Bogen nachzuzeichnen und die Breite der Möglichkeiten in der täglichen Praxis der Palliativmedizin aufzuzeigen.

Den Teilnehmern des Kurses ist herzlich zu gratulieren, dass sie auch in diesem 3. Kurs eine qualitativ hochwertige Sammlung von schwierigen Praxisfällen dargestellt haben und sich nicht scheuten, auch kritische Fragen und heikle Probleme aufzugreifen. $\mathrm{Zu}$ danken ist den vielen Vortragenden des Kurses, den Mitgliedern des wissenschaftlichen Beirates und dem Organisationskomitee sowie den Mitarbeitern des Springer-Verlages für die vielfältigen Hilfen bei der Erstellung dieses Heftes, das hoffentlich viele interessierte Leser finden wird.

\section{Lehrgangsleitung}

Univ.-Prof. Dr. Johannes G. Meran (wissenschaftlicher Leiter), Krankenhaus der Barmherzigen Brüder, Wien

Univ.-Prof. Dr. Herbert Watzke, Medizinische Universität Wien

Dr. Annette Henry, CS-Hospiz Rennweg, Wien

Dr. Dietmar Weixler MAS (palliative care), Landesklinikum Waldviertel Horn, PKD

\section{Beirat}

Univ. Prof. Dr. Paul Aiginger, MR Dr. Gerhard Aigner (BMGF), Univ. Prof. Dr. Alexander Gaiger, Univ. Prof. Dr. Günther Gastl, Univ. Prof. Dr. Erny Gillen (Caritas Europa), Univ. Prof. Dr. Richard Greil, Univ. Prof. Dr. Wilfried Ilias, Univ. Prof. Dr. Meinolf Karthaus, Univ. Prof. Dr. Heinz Ludwig, Univ. Prof. Dr. Dieter Lutz, Univ. Prof. Dr. Christine Marosi, Univ. Prof. Dr. H.C. Müller Busch, Univ. Prof. Dr. Friedemann Nauck, Univ. Prof. Dr. Martin Pecherstorfer, Univ. Prof. Dr. Michael Preitschopf, Univ. Prof. Dr. Lukas Radbruch, Univ. Prof. Dr. Heinrich Resch, Univ. Prof. Dr. Hellmut

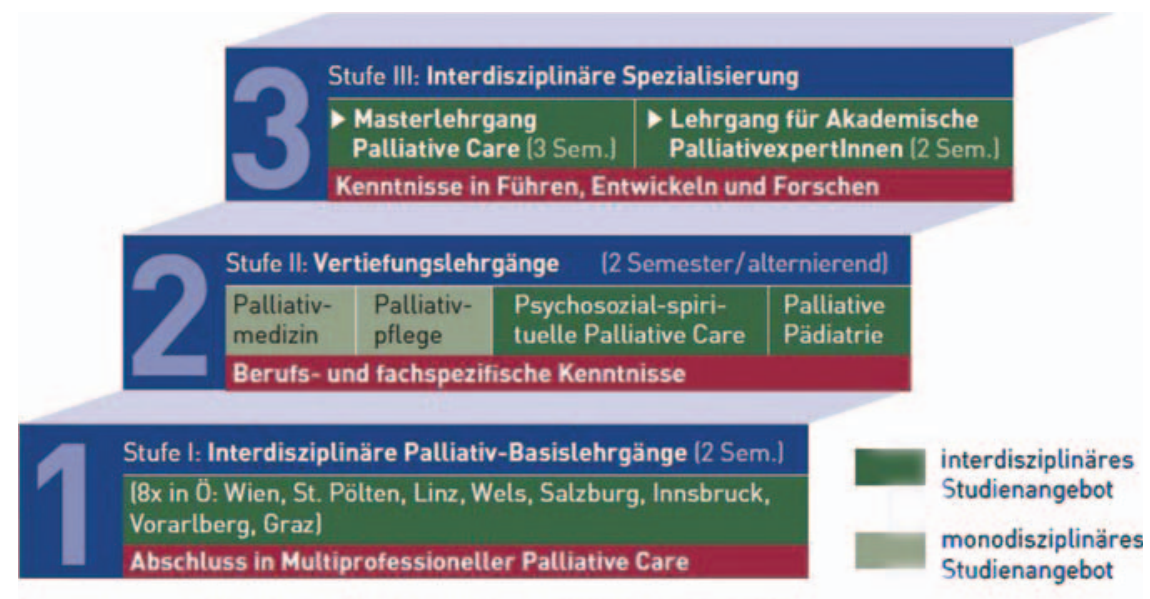

Voraussetzung für Master- bzw. Expertinnenabschluss sind mindestens 4 Semester interdisziplinäres Studium

Studienangebote in Stufe I und Stufe II können auch einzeln als Fortbildung besucht werden 
Samonigg, Rektor Dr. Wolfgang Schütz (MedUniWien), Univ. Prof. Dr. Mathias Volkenandt.

\section{Vortragende}

Univ. Prof. Dr. Herbert Watzke, Dr. Annette Henry, Univ. Prof. Dr. Johannes G. Meran, Dr. Dietmar Weixler MAS (palliative care), Peter Fässler-Weibel, Dr. Harald Retschitzegger MAS (palliative care), DI Dr. Heinz Lahrmann, Prim. Dr. Walter Döller, Dr. Wolfgang Hartmann, Univ. Prof. Dr. Andreas Sandner-Kiesling, Univ. Prof. Dr. Friedemann Nauck, Univ. Prof. Dr. Lukas Radbruch, Dr. Tilly Egger, Univ. Prof. Dr. Michael Binder, Prim. Dr. Georg Psota, Dr. Ines Eberl, Dr. Gertrude Bogyi, Univ. Prof. Dr. Rudolf Likar, Univ. Prof.
Dr. Meinolf Karthaus, Univ. Prof. Dr. HC Müller-Busch, Univ. Prof. Dr. Leonhart Thun. Hohenstein, Dr. Johann Zoidl, Prim. Dr. Roland Kunz, Dr. Peter Reichenpfader, Univ. Prof. Dr. Anton Stift, Dr. Maria Kletecka-Pulker, Univ. Prof. Dr. Wilfied Ilias, Univ. Prof. Dr. Jürgen Osterbrinck, Univ. Prof. Mag. Mag. DD Dr. Clemens Sedmak.

Der Vertiefungslehrgang/Stufe II im Rahmen des Universitätslehrganges Palliative Care findet von Jänner 2010 bis Juni 2011 (2 Semester) statt. Weitere Informationen finden Sie unter: www.hospiz.at.

\section{Interessenskonflikt}

Es besteht kein Interessenskonflikt. 